Nervenarzt 2008 · 79:543-557 DOI 10.1007/s00115-008-2411-8

Online publiziert: 16. Februar 2008

(c) Springer Medizin Verlag 2008

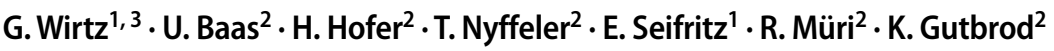

${ }^{1}$ Universitäre Psychiatrische Dienste Bern, Universitätsklinik

für Klinische Psychiatrie, Bern

${ }^{2}$ Abteilung für neuropsychologische Rehabilitation, Neurologische Klinik der Universität, Inselspital Bern

${ }^{3}$ Fachklinik für Psychiatrie, Psychotherapie und Psychosomatik,

Klinik an der Lindenhöhe, Offenburg

\section{Psychopathologie des Ganser-Syndroms}

\section{Literaturübersicht und Falldiskussion}

Obwohl sich das Forschungsinteresse an dissoziativen Störungen in den letzten Jahren intensiviert hat und in diesem $\mathrm{Zu}$ sammenhang mehrere Störungsmodelle formuliert wurden, bleiben die ätiopathogenetischen Modelle hypothetisch [12, 32, 33]. Insbesondere gilt dies für das 1897 von Ganser beschriebene und nach ihm benannte „Ganser-Syndrom “ [7, 21], das in den aktuellen Klassifikationssystemen zu den dissoziativen Störungen gezählt wird $[14,44]$.

Das Kernsymptom ist das so genannte „Vorbeiantworten“ [21], auch als ,Vorbeireden“ [7] oder ,approximate answers“ [20] bzw. „near-miss answers" [25] bezeichnet. Neben diesem Kernsymptom gelten weitere Symptome als charakteristisch, wie etwa eine fluktuierende Bewusstseinsstörung, pseudoneurologische Phänomene, visuelle oder akustische Pseudohalluzinationen, dissoziative Fugue-Zustände, affektive Störungen in Form einer kindlich-läppischen Stimmungslage, depressive Verstimmtheit, pseudoepileptische Anfälle sowie verschiedene neuropsychologische Störungen wie Gedächtnislücken und Wahrnehmungsstörungen [21, 25, 47, 50]. Zusammenfassend wurde diese Symptomatik auch als „Pseudodemenz" bezeichnet [30].

Die Tab. 1 und 2 geben einen Überblick über die Publikationen, die in einer Medline-Suche bis $2006 \mathrm{zu}$ finden waren (• Tab. 1) und Übersichtsartikeln, die die publizierten Fallberichte bis zur Erstbeschreibung von Ganser zurückverfolgen (• Tab. 2).

Aufgrund des seltenen Auftretens lassen sich keine sicheren epidemiologischen Angaben etwa bezüglich Häufigkeit, Geschlecht (tendenziell häufiger bei Männern), Kultur oder zeitlicher Dauer (zwischen Tagen und Monaten; teilweise mit Fluktuationen jahrelang) machen [12]. Es zeigt sich allerdings, dass die Erkrankung sehr häufig mit zerebralen Schädigungen, insbesondere Schädel-Hirn-Traumen assoziiert ist.

Für die Annahme einer dissoziativen Störung spricht, dass die Erkrankung häufig unter traumatischen oder zumindest stark belastenden Bedingungen entsteht und dass Verlauf und Vielgestaltigkeit der Symptomatik durch eine umschriebene Hirnfunktionsstörung nur schwer erklärt werden können.

In der Diskussion um die Ätiopathogenese der Erkrankung fehlen systematische Untersuchungen. 0 Tab. 1 und 2 fassen in 151 Fällen Hinweise auf eine zerebrale Schädigung, Ergebnisse bildgebender Diagnostik und neuropsychologische Untersuchungen zusammen. Dabei fällt auf, dass häufig keine neurologische oder neuropsychologische Untersuchung erfolgt ist. Die wenigen vorhandenen Befunde deuten dabei auf eine Störung vor allem frontal-exekutiver Funktionen hin $[13,23,25,30]$. Kaum untersucht ist, inwiefern das „Vorbeiantworten“ als Ausdruck einer „nichtaphasischen Kommunikationsstörung" mit weitschweifiger, inkohärenter Sprache und Vernachlässigen von Kontextinformationen zu werten ist, wie sie bei präfrontalen und rechtshemisphärischen Läsionen beschrieben ist $[16,22,40]$.

Somit bleibt trotz aller Versuche einer systematischen Charakterisierung das „Vorbeiantworten“ das zentrale psychopathologische Merkmal des GanserSyndroms. Dies bestätigt sich auch in unserem Fall.

Sowohl der mutmaßlich neurologischneuropsychologischen als auch psychiatrischen Ätiologie Rechnung tragend, besteht das Ziel der vorliegenden Arbeit darin, mittels der Ergebnisse der differenzierten psychiatrischen, neuropsychologischen und neurolinguistischen Untersuchung sowie der zerebralen Bildgebung zu klären, in welchem Zusammenhang das zentrale psychopathologische Symptom des "Vorbeiantwortens“ mit spezifischen frontal-exekutiven Hirnfunktionsstörungen stehen könnte.

\section{Fallbericht}

Wir berichten über eine 43-jährige Patientin (Erzieherin), die bis zum Zeitpunkt ihres Hirninfarktes als Hausfrau und Mutter (2 Söhne im Alter von 8 und 19 Jahren) tätig war. 
Tab. 1 Publikationen mit Angaben zu Fällen mit einem Ganser-Syndrom entsprechend einer Medline-Suche bis 2006

\begin{tabular}{|c|c|c|c|c|c|c|}
\hline Autor & Fälle & Erwachsene & $\begin{array}{l}\text { Kinder, } \\
\text { Jugendliche }\end{array}$ & $\begin{array}{l}\text { Hinweis auf zerebrale Schä- } \\
\text { digung }\end{array}$ & Bildgebung & $\begin{array}{l}\text { Neuropsychologische } \\
\text { Tests }\end{array}$ \\
\hline Tsoi [50] & 10 & $x$ & & Unklar & Keine & Keine \\
\hline Latcham et al. [33] & 1 & $x$ & & $\mathrm{Ja}$ & Keine & Keine \\
\hline Dabholkar [9] & 1 & & $x$ & Nein & Nein & Nein \\
\hline Schneider et al. [45] & 1 & & $x$ & Keine Angaben & Keine Angaben & Keine Angaben \\
\hline Adler et al. [1] & 1 & & $\mathrm{x}$ & Keine Angaben & Keine Angaben & Keine Angaben \\
\hline Heron et al. [25] & 1 & $x$ & & $\mathrm{Ja}(\mathrm{SHT})$ & $\mathrm{Ja}(\mathrm{CT})$ & $\begin{array}{l}\text { WAIS, BNT, COWA, TMT, } \\
\text { WCST, CVLT, WMS, MMPI }\end{array}$ \\
\hline Sigal et al. [47] & 15 & $\mathrm{X}$ & & $\begin{array}{l}\text { Ja in } 7 \text { Fällen ( } 6 \mathrm{SHT}, 1 \mathrm{CVI} \\
\text { rechts mit Hemiparese links) }\end{array}$ & Keine & Keine \\
\hline Apter et al. [6] & 2 & & $\mathrm{x}$ & Keine Angaben & Keine Angaben & Keine Angaben \\
\hline Hampel et al. [23] & 1 & $x$ & & Nein & $\mathrm{Ja}$ (EEG, CT, MRI) & MMSE \\
\hline Miller et al. [35] & 1 & & $x$ & $\mathrm{Ja}(\mathrm{SHT})$ & Keine Angaben & Keine Angaben \\
\hline Dalfen et al. [10] & 4 & $\mathrm{X}$ & & Ja (alle SHT) & $\begin{array}{l}\text { Ja in } 3 \text { Fällen (CT, } \\
\text { MRI, SPECT) }\end{array}$ & Nein \\
\hline Lee et al. [34] & 1 & $\mathrm{x}$ & & Keine Angaben & Keine Angaben & Keine Angaben \\
\hline Andersen et al. [5] & 1 & $x$ & & Keine Angaben & Keine Angaben & Keine Angaben \\
\hline Refaat et al. [43] & 1 & & $x$ & $\begin{array}{l}\text { Ja (Gilles-de-la-Tourette- } \\
\text { Syndrom) }\end{array}$ & Keine Angaben & Nein \\
\hline Deibler et al. [13] & 1 & $\mathrm{X}$ & & $\mathrm{Ja}(A I D S)$ & Ja (MRI) & $\begin{array}{l}\text { MMSE, TOMM, WAIS, WRAT, } \\
\text { MMPI }\end{array}$ \\
\hline $\begin{array}{l}\text { Ladowsky-Brooks et } \\
\text { al. [30] }\end{array}$ & 1 & $\mathrm{X}$ & & $\begin{array}{l}\text { Ja (Frontalhirnpathologie, } \\
\text { Ursache unklar) }\end{array}$ & Ja (MRI, SPECT) & $\begin{array}{l}\text { WAIS, Rey Figure (Copy + } \\
\text { Immediate Recall), WMS, } \\
\text { HVO, TOMM, WAB }\end{array}$ \\
\hline \multicolumn{7}{|c|}{$\begin{array}{l}\text { BNT Boston Naming Test; COWA Controlled Oral Word Association Test; CT Computertomographie; CVI zerebrovaskulärer Insult; CVLT California Verbal Learning Test; EEG } \\
\text { Elektroenzephalographie; HVO Hooper Visual Organization Test; MMPI Minnesota Multiphasic Personality Inventory; MRI Magnetic Resonance Imaging; SHT Schädel-Hirn- } \\
\text { Trauma; SPECT Single Photon Emission Tomography; TOMM Test of Memory and Malingering; TMT Trail-Making-Test; WAB Western Aphasia Battery; WAIS Wechsler Adult } \\
\text { Intelligence Scale; WCST Wisconsin Card Sorting Test; WMS Wechsler Memory Scale; MMSE Mini Mental State Examination; WRAT Wide-Range Achievement Test. }\end{array}$} \\
\hline
\end{tabular}

Bis zu dem vor Aufnahme plötzlich aufgetretenen rechtsseitigen sensomotorischen Hemisyndrom zeigten sich in der Vorgeschichte keine Hinweise auf eine psychiatrische Erkrankung, Drogen- bzw. Alkoholkonsum oder neurologische Erkrankungen. Die initiale diffusions- und perfusionsgewichtete magnetresonanztomographische (MRI-)Untersuchung zeigte eine Infarzierung im Stromgebiet der linken Arteria cerebri media (MCA). Bezüglich der Versorgungsgebiete der leptomeningealen Äste der MCA (vgl. [49]) konnten Diffusions- bzw. Perfusionsstörungen im gesamten Gyrus frontalis medius und inferior inkl. der Insula, im Gyrus postcentralis sowie dem superioren und inferioren parietalen Lobulus (Gyrus angularis weniger betroffen) festgestellt werden. Bezüglich der Versorgungsgebiete der perforierenden Äste der MCA lagen ferner Diffusions- bzw. Perfusionsstörungen des Ncl. caudatus, der Capsula interna, dem Putamen, Klaustrum bis zum Globus pallidus reichend vor. Ei- ne MR-Angiographie zeigte einen Verschluss des linken distalen M1-Segmentes der MCA aufgrund einer Dissektion der linken Arteria carotis interna unmittelbar nach der Bifurkation.

Aufgrund eines Ödems der linken Hemisphäre wurde eine linkshemisphärische Kraniotomie durchgeführt. Ein KontrollCT zeigte einen Infarkt im linken Mediastromgebiet ohne hämorrhagische Transformation (• Abb. 1).

Ein EEG zeigte rechtshemisphärisch eine normale Grundaktivität, linkshemisphärisch einen kontinuierlich ausgeprägten frontotemporalen Herdbefund ohne epilepsiespezifische Potenziale.

Im weiteren klinischen Verlauf blieben die unten beschriebenen Defizite während eines stationären Rehabilitationsaufenthaltes (3 Monate) und einer weiteren Langzeitrehabilitation (3 Monate) trotz intensiver Neurorehabilitation unverändert. Die Patientin lebt heute mit intensiver ambulanter Betreuung zu Hause.

\section{Klinische Auffälligkeiten}

Im Gespräch machte die Patientin bei flüssiger und unauffälliger Spontansprache sowie erhaltenem Verständnis der Fragen bzw. Instruktionen falsche bis völlig unplausible Angaben auf einfache Fragen (z. B. sei sie Lehrerin an einer Ergotherapieschule; ihre Kinder seien 2008 und 2009 geboren). Die spontanen Antworten erfolgten ohne jegliches Zögern oder Bemühen um Richtigkeit.

\section{Neuropsychologische Befunde}

Die Durchführung einer standardisierten neuropsychologischen Untersuchung war aufgrund der zuvor beschriebenen Auffälligkeiten deutlich erschwert. Die folgenden Resultate beziehen sich auf Untersuchungsergebnisse, die über mehrere Sitzungen nahezu konstant waren. 
Hier steht eine Anzeige.

算 Springer 
Nervenarzt 2008 · 79:543-557 DOI 10.1007/s00115-008-2411-8

(c) Springer Medizin Verlag 2008

G. Wirtz · U. Baas · H. Hofer · T. Nyffeler · E. Seifritz · R. Müri · K. Gutbrod

Psychopathologie des Ganser-Syndroms. Literaturübersicht und Falldiskussion

\section{Zusammenfassung}

Das Kernsymptom des Ganser-Syndroms besteht im „Vorbeiantworten" auf einfache Fragen. Die Ursache dieses seltenen Syndroms ist unklar. Aktuelle Klassifikationssysteme zählen es zu den dissoziativen Störungen, wobei eine psychogene Ursache der Symptome angenommen wird. Anhand einer Literaturrecherche $(n=151)$ wird jedoch gezeigt, dass das Ganser-Syndrom sehr häufig mit Hirnverletzungen assoziiert ist, wobei detaillierte bildgebende, neuropsychologische und neurologische Untersuchungen weitgehend fehlen. Wir stellen eine rechtshändige Patientin mit einem Ganser-Syndrom nach einem großen linkshemisphärischen Mediainfarkt vor. Die detaillierte neuropsychologische Un- tersuchung zeigte eine untypische Lateralisierung kognitiver Funktionen mit einer sog. gekreuzten Nichtaphasie und ausgeprägten frontal-exekutiven Funktionsstörungen. Unter Berücksichtigung sowohl der psychiatrischen als auch der neuropsychologischen Aspekte wird diskutiert, in welchem Zusammenhang das psychopathologische Symptom des "Vorbeiantwortens" mit spezifischen frontal-exekutiven Hirnfunktionsstörungen stehen könnte.

Schlüsselwörter

Ganser-Syndrom · Dissoziative Störung ·

Gekreuzte Nichtaphasie · Frontal-exekutive Störungen

\section{Psychopathology of Ganser's syndrome. Literature review and case report}

\section{Summary}

The key feature of Ganser's syndrome includes approximate answers to simple questions. The cause of this rare syndrome remains uncertain. Current classification systems categorise it as a dissociative disorder, the symptoms of which are judged as psychogenic in origin. Our review of the literature $(n=151)$ demonstrates however that Ganser's syndrome is frequently associated with brain injury, although detailed imaging, neuropsychological, and neurological data of this for the most part do not exist. We describe a right-handed patient with Ganser's syndrome after a large left-hemispheric mid- dle cerebral artery infarction. Detailed neuropsychological examination showed atypical lateralisation of cognitive functions with socalled crossed nonaphasia and pronounced frontal-executive dysfunctions. Regarding both psychiatric and neuropsychological aspects, we discuss how the key feature of approximate answers may be associated with frontal-executive cerebral dysfunctions.

Keywords

Ganser's syndrome - Dissociative disorder . Crossed nonaphasia $\cdot$ Frontal-executive dysfunction

\section{Händigkeit}

Eigen- und fremdanamnestisch bestand prämorbid reine Rechtshändigkeit. In der Herkunftsfamilie sind keine Linkshänder bekannt.

\section{Prüfung auf Aggravations- bzw. Simulationsverdacht}

Aufgrund des ungewöhnlichen Antwortverhaltens, der Diskrepanz zwischen Testund Alltagsverhalten und da der Aspekt von Simulation und Aggravation im $\mathrm{Zu}$ sammenhang mit dem Ganser-Syndrom diskutiert wird, wurde diesbezüglich ein Test durchgeführt [26], der unauffällig war.

\section{Sprache, Sprechen und Kommunikation}

Sprachdominanz. Aufgrund des Schädigungsprofils hätte man bei dieser rechtshändigen Patientin eine schwere Aphasie erwartet. Das Fehlen einer aphasischen Symptomatik ließ vermuten, dass bei der Patientin eine atypische rechtshemisphärische Sprachdominanz vorliegt (vgl. [4]). $\mathrm{Da}$ sie eine funktionelle Magnetresonanztomographie ablehnte, führten wir den dichotischen Hörtest von Hugdahl und Mitarbeitern [28] durch. Die Patientin erreichte einen Lateralitätsindex von -93 , was für eine rechtshemisphärische Sprachdominanz spricht.

Sprachsystematische Untersuchung des Sprechens und der Sprache. Es wurde die „Kurze Aphasieprüfung“ (KAP) [31] durchgeführt. Die Ergebnisse finden sich in - Tab. 3.

In der Spontansprache zeigte sich bei relativ schneller Sprechgeschwindigkeit eine vorwiegend affektive Aprosodie mit monotoner, kaum nivellierter Sprechweise ohne Zeichen einer Dysarthrophonie. Phonematik und Syntax waren unauffällig. Ebenso waren in der Spontansprache keine offensichtlichen Einbußen im Sprachverständnis erkennbar. Die sprachlichen Äußerungen waren geprägt durch häufige Iterationsphänomene in Form von Perseverationen und Stereotypien sowie durch das Auftreten von - rein formal zu klassifizierenden - semantischen Paraphasien (z. B. „leicht“ statt „schwer“). Wortfindungsstörungen im engeren Sinne traten praktisch nie auf. 
Hier steht eine Anzeige.

算 Springer 


\begin{tabular}{|c|c|c|c|c|c|c|}
\hline Autor & Fälle & Erwachsene & $\begin{array}{l}\text { Kinder, } \\
\text { Jugendliche }\end{array}$ & $\begin{array}{l}\text { Hinweis auf zere- } \\
\text { brale Schädigung }\end{array}$ & Bildgebung & $\begin{array}{l}\text { Neuropsychologische } \\
\text { Tests }\end{array}$ \\
\hline Ganser [21] & 4 (3 Kasuistiken) & $\mathrm{X}$ & & Ja in 2 Fällen (SHT) & Keine & Keine \\
\hline Assion[7] & $\begin{array}{l}142 \text { (alle weltweit veröf- } \\
\text { fentlichten Kasuistiken } \\
\text { von } 1888 \text { - 1997) }\end{array}$ & $x$ & $\mathrm{X}$ & $\begin{array}{l}\text { Keine detaillierten } \\
\text { Angaben }\end{array}$ & $\begin{array}{l}\text { Keine detaillierten } \\
\text { Angaben }\end{array}$ & $\begin{array}{l}\text { Keine detaillierten } \\
\text { Angaben }\end{array}$ \\
\hline Assion et al. [8] & $\begin{array}{l}33 \text { (alle Patienten mit } \\
\text { dem Hinweis auf körper- } \\
\text { liche Auffälligkeiten von } \\
\text { 1888-1995) }\end{array}$ & $x^{a}$ & $x^{a}$ & $\begin{array}{l}\text { Mindestens } 26 \\
\text { (SHT, Krampfanfälle, } \\
\text { Entzündungen etc.) }\end{array}$ & $\begin{array}{l}\text { Ja, keine konkreten } \\
\text { Angaben }\end{array}$ & $\begin{array}{l}\text { Keine detaillierten } \\
\text { Angaben }\end{array}$ \\
\hline Dammann [12] & $\begin{array}{l}\text { Ca. } 43 \text { ausführlich be- } \\
\text { schriebene Fälle bis 1984, } \\
\text { danach eine größere } \\
\text { Fallsammlung: Sigal et } \\
\text { al. } 1992\end{array}$ & $x$ & $x$ & $\begin{array}{l}\text { Keine detaillierten } \\
\text { Angaben }\end{array}$ & Keine Angaben & Keine Angaben \\
\hline
\end{tabular}

Nachsprechen, Reihensprechen, Abschreiben, Benennen (Wortfindung) und auditives Sprachverständnis waren unauffällig.

Diktatschreiben und Lesen waren schwer vermindert, wobei die typischen Symptome einer Agraphie und Alexie (z. B. phonematische oder semantische Paragraphien/Paralexien) fast vollständig fehlten. Es zeigten sich hingegen fast ausschließlich Fehler, die als Ausdruck exekutiver Störungen in Form einer hohen Perseverationsanzahl und Fortsetzungsfehler zu interpretieren sind, d. h. fast immer korrekter Beginn des Zielreizes und dann ein Abgleiten in perseverative Fehler (z. B. „Eieee“ statt „Eis“, „zahlungsfähig“ statt „Zahl“). Entsprechend diesen Problemen war auch das „Lesesinnverständnis“ schwer vermindert.

Kommunikationsverhalten. Im pragmatischen Verhalten der Patientin, eingeschätzt nach dem pragmatischen Protokoll nach Prutting u. Kirchner [41], zeigten sich vor allem Auffälligkeiten im Sinne einer Negativsymptomatik: Die Patientin initiierte selten einen kommunikativen Akt. Konkrete oder auch offene Fragen wurden meist nur mit Ja, Nein oder „ich weiß nicht“ beantwortet. In Bezug auf die Wortwahl zeigte sich eine geringe Spezifizität. Stilvariationen fanden kaum statt. Die Prosodie und der Redefluss waren vermindert, während die Verständlichkeit, die Stimmintensität und -qualität keine Auffälligkeiten aufwiesen. Mimische und gestische Elemente wurden kaum eingesetzt (Amimie), der Blickkontakt war adäquat.
Auf der diskursiven Ebene des Kommunikationsverhaltens (vgl. [15]) ergaben sich hinsichtlich der Hauptthemen der Erzählungen (makropropositionale Ebene) Auffälligkeiten wie Themensprünge und -abbrüche, die Kohärenz war vermindert, die Erzählungen waren unlogisch, somit war auch der Informationsgehalt und die Plausibilität des Erzählten deutlich reduziert. Auf mikropropositionaler Ebene fielen vor allem fehlende Verknüpfungen zwischen den Sätzen und unklare Referenzen auf. Qualitativ wurden die Äußerungen rasch, ohne Zögern vermittelt, wobei man klinisch den Eindruck gewann, dass die Patientin selbst kaum am Sinngehalt des Gesagten zweifelte.

\section{Überprüfen der „,klassischen“ Symptome eines Ganser-Syndroms mittels der Be- antwortung einfacher Fragen. Das Kar-} dinalsymptom des Ganser-Syndroms ist das so genannte „Vorbeiantworten“. Um diesen Aspekt zu objektivieren, wurden 10 einfache Fragen zum allgemeinen Wissen formuliert (z. B. „Wie viele Beine hat ein Pferd?"). In der ersten Bedingung wurde die freie Antwort erfasst. In der zweiten Bedingung wurden vier Antwortmöglichkeiten verbal-akustisch vorgegeben, die richtige Antwort sollte benannt werden. Unter beiden Bedingungen wurden nur 3/10 korrekte Antworten gegeben, wobei wie beim Ganser-Syndrom zu erwarten die Falschantworten nur knapp von den korrekten Antworten abwichen (z. B. Wie viel Beine hat ein Pferd? Antwort: 5).

\section{Zahlenverarbeitung und Arithmetik}

In der Zahlenverarbeitung zeigten sich schwerste Störungen ähnlich den schriftsprachlichen Leistungen (Perseverationen, „Vorbeiantworten“). Auch einfachste Grundrechenarten, z. B. 3+4 (Antwort 6) konnten weder schriftlich noch mündlich gelöst werden.

\section{Praxie}

Bei der klinischen Apraxieprüfung zeigte sich ein ausgeprägtes Perseverationsverhalten, welches im Rahmen der schweren Störungen exekutiver Funktionen zu werten ist. Dafür spricht auch, dass das Verhalten bei realem Objektgebrauch und einfachen Alltagsverrichtungen (z. B. Hygiene) weitgehend unauffällig war.

\section{Exekutive Funktionen}

Ein großer Teil exekutiver Verfahren konnte nicht durchgeführt werden, da die Patientin bereits beim Verständnis der Instruktion scheiterte (z. B. Stroop-Test, Links-Probe, Tower-Aufgaben, 20 Questions) oder sich im Lauf der Testdurchführung nicht mehr an die Instruktion hielt. So waren die Leistungen bei der Untersuchung verschiedener Fluenzmaße durch Perseverationen und Regelbrüche geprägt (etwa bei der Aufgabe Wörter mit dem Anfangsbuchstaben S zu nennen: „Sonnenblume, Geranium, Sabine, Ventilator, Therapie...").

\section{Gedächtnis}

Kurzzeit- bzw. Arbeitsgedächtnis (vgl. [24]). Bei der verbalen Merkspanne fiel 
Hier steht eine Anzeige.

算 Springer 


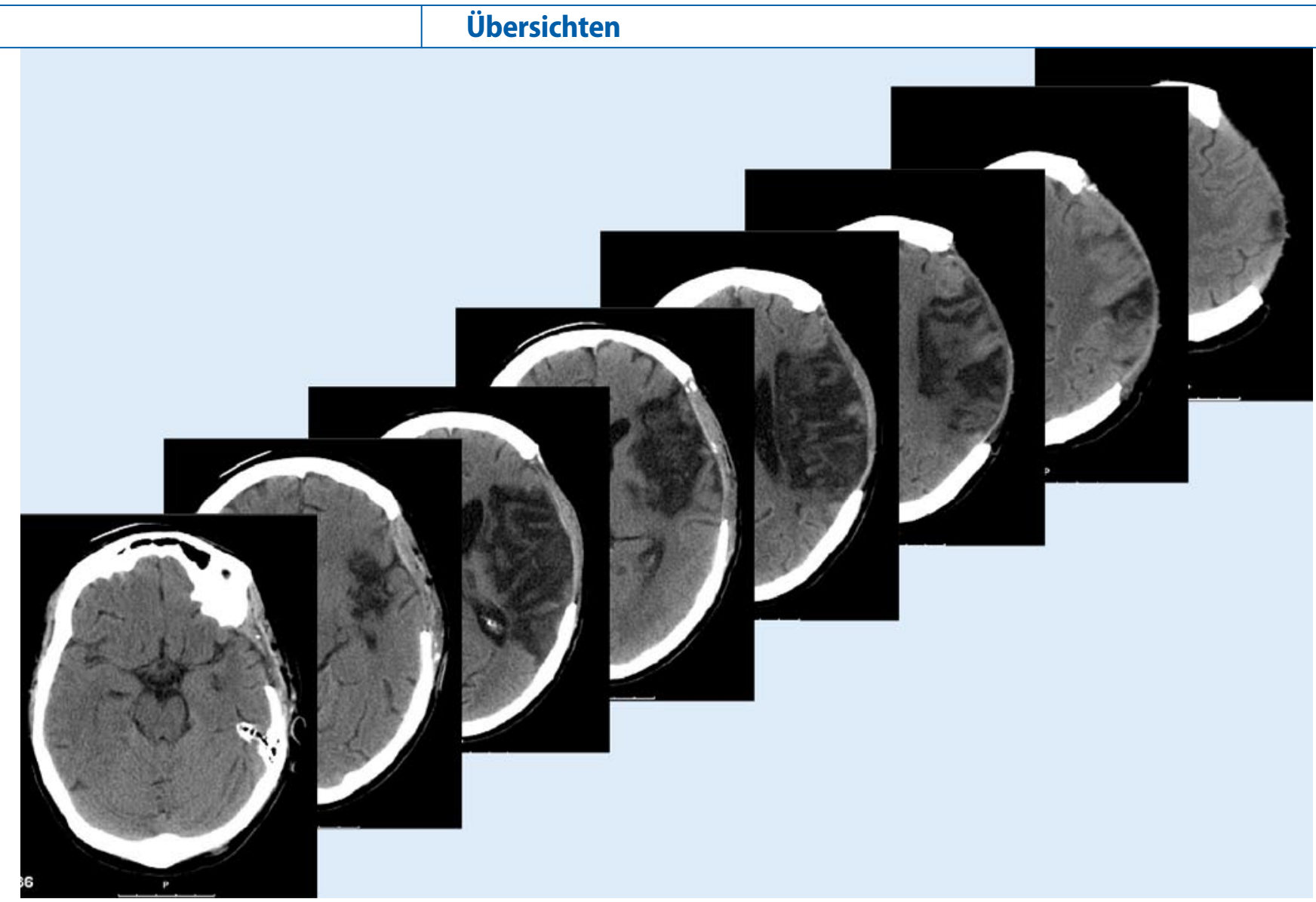

Abb. 1 Die computertomographischen Bilder der Patientin zeigen die hypodense Läsion im Stromgebiet der linken Arteria cerebri media, der Gyrus praecentralis und die posterotemporale Region sind ausgespart (vgl. Text)

auf, dass die richtig wiedergegebenen Zahlenreihen immer um eine weitere Zahl ergänzt wurden, so dass die Patientin formal auf einen Punktwert von 4 (T-Wert: 29) kam. Ohne Berücksichtigung dieser zusätzlich wiedergegebenen Zahl wurde ein Punktwert von 9 (T-Wert: 57) erreicht. Die visuell-räumliche Merkspanne war mit einem Punktwert von 4 (T-Wert: 21) erheblich vermindert.

Episodisches Gedächtnis. Das episodische Gedächtnis wurde in der verbalen Modalität mit dem Subtest „Wortliste" aus dem CERAD (vgl. [37]) und in der nonverbalen Modalität mit dem „Rey Visual Design Learning Test“ (vgl. [48]) geprüft. Beim Lernen, Abrufen und Wiedererkennen verbaler Informationen (Wortliste mit 10 Items) ergaben sich Beeinträchtigungen beim Lernen, insbesondere jedoch aber beim Abruf (1 von 10 Items) mit vielen Intrusionen (Nennen von Wörtern, die nicht dargeboten wurden) und im Wiedererkennen (10 richtige Ja-, jedoch keine richtige Nein-Antwort, d. h. es bestand eine ausgeprägte „Ja-Sage-Tendenz").

Schwer beeinträchtigt war auch die Lern- und längerfristige Behaltensfähigkeit von nonverbalen Informationen, wobei sich ebenfalls eine ausgeprägte Perseverationstendenz zeigte.

\section{Sehen, visuelle Wahrnehmung, visuell-räumliche und -konstruktive Leistungen}

Es fanden sich keine Hinweise auf Einschränkungen des Nah- und Fernvisus, des Gesichtsfeldes oder auf Beeinträchtigungen im Bereich elementarer visueller Wahrnehmungsleistungen (z. B. Formund Farbwahrnehmung). Allerdings konnte ein hemispatialer Neglekt bzw. Extinktionsphänome nach rechts nachgewiesen werden sowie Einbußen visuokonstruktiver Fähigkeiten.

\section{Aufmerksamkeitsfunktionen}

Klassische Verfahren zur Aufmerksamkeitsprüfung konnten nicht durchgeführt werden. Untersuchbar war die Reaktions- fähigkeit auf einfache optische Reize, als Maß für das allgemeine Aktivierungsniveau („alertness"). Hierbei zeigten sich keine von der Altersnorm abweichende Leistungen.

\section{Psychiatrische Befunde}

Psychopathologischer Befund. Zentral war hier durchgehend das „Vorbeiantworten“. Affektiv wirkte die ruhige, eher gehemmte Patientin wenig moduliert, unbeteiligt, teils unerklärlich lächelnd.

Zur Überprüfung der Verdachtsdiagnose eines Ganser-Syndroms wurde das strukturierte klinische Interview nach $D S M-I V$ [51] durchgeführt. Dabei war das Kriterium „Annäherungsweise richtige Antworten auf Fragen, wenn dies nicht mit einer dissoziativen Amnesie oder dissoziativen Fugue einhergeht" erfüllt.

\section{Diskussion}

Aufgrund der Lokalisation und der Größe der Ischämie wäre bei unserer Patien- 
Hier steht eine Anzeige.

算 Springer 
Tab. 3 Testergebnisse „Kurze Aphasieprüfung

\begin{tabular}{|c|c|c|c|}
\hline Subtest & Rohwert & T-Wert ${ }^{a}$ & Prozentrang $^{a}$ \\
\hline Token-Test & $0 / 10$ & 42 & 20,1 \\
\hline Nachsprechen & $10 / 10$ & 73 & 100 \\
\hline Reihensprechen & $3 / 3$ & - & - \\
\hline Abschreiben & $5 / 6$ & - & - \\
\hline Diktatschreiben & $2 / 10$ & 43 & 23,5 \\
\hline Lautlesen & $2 / 10$ & 46 & 33,8 \\
\hline Sprachverständnis & $10 / 10$ & 73 & 100 \\
\hline Benennen & $9 / 10$ & 66 & 94,5 \\
\hline Lesesinnverständnis & $0 / 10$ & 45 & 32,5 \\
\hline Gesamt & $33 / 70$ & 49 & 45,7 \\
\hline \multicolumn{4}{|c|}{$\begin{array}{l}\text { aDie T-Werte und Prozentränge beziehen sich nicht auf eine gesunde Normalpopulation wie dies üblicherweise } \\
\text { in der Neuropsychologie der Fall ist, sondern auf ein Standardisierungskollektiv von } 162 \text { aphasischen Patienten, } \\
\text { d. h. die Werte geben die Schwere der aphasischen Symptomatik an. Ein Prozentrang von } 20 \text { in einem bestimm- } \\
\text { ten Subtest (entspricht ungefähr einem T-Wert von 40; T-Werte haben einen Mittelwert von } 40 \text { und eine Stan- } \\
\text { dardabweichung von 10) bedeutet, dass } 80 \% \text { aller aphasischen Patienten bei diesem Test besser abschneiden. }\end{array}$} \\
\hline
\end{tabular}

tin, ein rechtseitiges sensomotorisches Hemisyndrom, allenfalls ein rechtsseitiger Gesichtsfeldausfall, eine Dysarthrie, Störungen frontal-exekutiver Funktionen und vor allem eine schwere Aphasie zu erwarten gewesen $[38,42]$. Tatsächlich findet sich ein rechtsseitiges sensomotorisches Hemisyndrom, neuropsychologisch zeigen sich quantitativ mit sehr wenigen Ausnahmen kognitive Minderleistungen in sämtlichen geprüften Domänen, diese sind jedoch qualitativ als direkte oder indirekte Auswirkung einer frontal-exekutiven Funktionsstörungen in der Planung, Steuerung, Ausführung und Kontrolle kognitiver Prozesse zu werten. Die Interpretation der kognitiven Minderleistungen als Ausdruck frontal-exekutiver Funktionsstörungen wird neuroradiologisch durch die ausgedehnte präfrontale dorsolaterale und ausgedehnte Läsion der „frontal-subcortical circuits" (vgl. [2]) gestützt.

Im Gegensatz zur erwarteten Symptomatik stellt sich allerdings keine Aphasie ein. Es zeigt sich auch nicht das seltene neuropsychologische Syndrom einer so genannten gekreuzten Nichtaphasie, d. h. das Fehlen einer Aphasie, aber Vorliegen von ausschließlich visuell-räumlichen und anderen typischen rechtshemisphärischen Defiziten bei einer rechtshändigen Patientin mit einer linkshemisphärischen Läsion $[4,17,29]$. Vielmehr tritt klinisch das Phänomen des „Vorbeiantwortens“ in den Vordergrund - das psychopathologisch als Leitsymptom des Ganser-Syndroms zu klassifizieren ist.

\section{Neuropsychologische Differenzialdiagnostik}

Während der Behandlung zeigte die Patientin nie Anzeichen eines Verwirrtheitszustandes mit Störung des Bewusstseins und der Orientierung, visuelle Halluzinationen, Unruhe, vegetative Symptome etc. Auch zeigten sich zu keinem Zeitpunkt Symptome einer spontanen Konfabulation mit Realitätsverlust [46].

Die zentrale Frage im vorliegenden Kontext ist jedoch, inwiefern das „Vorbeiantworten“ auch als aphasisches Symptom im Sinne einer „semantischen Paraphasie“ bzw. von Wortfindungsstörungen im weiteren Sinne interpretiert werden kann. Obgleich der Eindruck solcher scheinbarer „semantischer Paraphasien“ oder Wortfindungsstörungen entstand, ließen sie sich testpsychologisch nicht nachweisen. Allein diese Tatsache (Wortfindungsstörungen gelten als das Kardinalsymptom aller Aphasiesyndrome) und dass entsprechend unserem Wissen semantische Paraphasien nie als alleiniges aphasisches Symptom bei Aphasien auftreten [27], spricht dagegen, dass das „Vorbeireden“ einzig als Ausdruck einer semantischen Paraphasie zu werten ist. Darüber hinaus wäre aufgrund der linkshemisphärischen Läsionen bei dieser rechtshändigen Patientin am ehesten eine Broca-, eine transkortikal-motorische, eine Leitungs- oder gar eine globale Aphasie zu erwarten gewesen (vgl. [3, 11, 18, 36]). Bei keinem dieser Syndrome sind semantische Paraphasien die sprachlichen Leitsymptome [27].
Insgesamt scheint aufgrund der vorliegenden neuropsychologischen und bildgebenden Daten bei unserer Patientin eindeutig eine sehr untypische hemisphärische Dominanz kognitiver Funktionen vorzuliegen: Es handelt sich nicht um das typische Bild eine gekreuzten Nichtaphasie mit umgekehrter hemisphärischer Dominanz. Vielmehr sind durch die ausgeprägte linkshemisphärische Läsion sowohl klassische linkshemisphärische Funktionen (Lesen, Schreiben, Akalkulie, Apraxie, verbale Gedächtnisprozesse) als auch klassische rechtshemisphärische Funktionen (nonverbale Fluenz, nonverbale Gedächtnisprozesse, visuelle Extinktion, visuell-räumliche Leistungen) betroffen. Frontal-exekutive Funktionsstörungen determinieren entsprechend unserer Beurteilung die klinische Symptomatik. Aus der Literatur ist uns bei den gekreuzten Nichtaphasien kein einziger Fall bekannt, der ein ähnliches Symptom des „Vorbeiredens“ gezeigt hätte. Zwar können letztendlich im vorliegenden Fall semantische Paraphasien als Ausdruck des „Vorbeiredens" nicht vollkommen ausgeschlossen werden, sie erscheinen jedoch aufgrund der testpsychologischen Befunde und der Art der „sprachlichen Fehlleistungen“ dafür nicht typisch.

\section{Psychiatrische Differenzialdi- agnostik und Phänomenologie}

Die Bewertung aus psychiatrischer Perspektive folgt den Überlegungen, welcher diagnostischen Kategorie das psychopathologische Syndrom zuzuordnen ist und wie das „Vorbeiantworten “ phänomenologisch eingeordnet werden kann.

\section{Differenzialdiagnostik}

Aufgrund der strukturellen zerebralen Schädigung ist nach ICD-10 eine Einordnung entsprechend Foo-Fo9 (organische, einschließlich symptomatische psychische Störungen) nahe liegend. Obwohl eine ausführliche neuropsychologische Diagnostik durchgeführt wurde, gelingt es in unserem Fall aber nur zum Teil, eine eindeutige $\mathrm{Zu}$ ordnung des funktionellen Störungsmusters zur strukturellen Hirnschädigung herzustellen. Im Vordergrund steht dabei die Störung der exekutiven Funktionen, weshalb das psychopathologische Syndrom 
Hier steht eine Anzeige.

算 Springer 
mit einer Demenz in Verbindung gebracht werden könnte. Das Alter, die eindeutige Krankheitsursache (plötzlich auftretende Ischämie) wie auch das neuropsychologische Befundprofil sprechen jedoch gegen eine Demenzdiagnose. So ist es nahe liegend eine „organische dissoziative Störung“ (ICD-10: Fo6.5) zu diagnostizieren, die die Einordnung des zentralen psychopathologischen Symptoms, nämlich des „Vorbeiantwortens“ zulässt.

Auf diesem Wege gelangt man zur Diagnose eines als organisch einzuordnenden Ganser-Syndroms. Diese Einordnung bezieht sich auf das Kernsymptom des Ganser-Syndroms, denn obwohl damit der Symptomkomplex reduziert wird, erscheint es legitim, das Syndrom aufgrund seines zentralen Phänomens zu beschreiben, da die begleitenden Symptome als fakultativ und wechselhaft beschrieben werden.

\section{Phänomenologie}

Abzugrenzen ist der in der Psychopathologie übliche Gebrauch des auch als „Danebenreden“ gekennzeichneten „Vorbeiredens“, was als formale Denkstörung verstanden wird, mit Antworten, die nicht zur gestellten Frage passen. Diese Begriffsbildung bezeichnet eine spezifisch schizophrene Psychopathologie mit einem Begriffszerfall, bei dem Begriffe ihre feste Bedeutung und ihre scharfe Abgrenzung verlieren. Durch diese Veränderung des Denkens und sprachlichen Ausdrucks erscheinen die Äußerungen der schizophren Erkrankten unbestimmt, verschwommen und teilweise bizarr [39]. Diese Beschreibungen des Phänomens im Sinne einer psychotischen Psychopathologie muss vom „Vorbeiantworten“ im Sinne Gansers unterschieden werden. Der Unterschied liegt dabei in der Willkür von Antworten, die beim psychotischen Patienten ein Spektrum zwischen völlig zusammenhanglosen Antworten bis hin zu knapp falschen Antworten aufweisen, während bei Ganser-Patienten, die knapp daneben liegenden Antworten das „Vorbeiantworten" ausmachen.

\section{Neurobiologische Grundlage des Phänomens}

Die Ursachen und neurobiologischen Grundlagen des Ganser-Syndroms sind unbekannt. In der Diskussion über die Ätiopathogenese der Störung werden unterschiedliche Hypothesen herangezogen [12]: Es könnte sich um atypische dissoziative Zustände, den dissoziativen Amnesien entsprechend, handeln oder um bewusst oder heimlich vorgetäuschte Störungen bei vorbestehenden $z$. B. histrionischen oder dissozialen Persönlichkeitsstörungen mit komorbiden hirnorganischen Störungen. Genauso könnte es sich aber auch um eine hirnorganische Störung per se handeln, basierend auf der Tatsache, dass die Mehrheit der in der Literatur beschriebenen Fälle eine Hirnschädigung unterschiedlicher Art aufweist. Leider fehlen in der Literatur bisher detaillierte Untersuchungen dieser Hypothesen, insbesondere die Angaben zu hirnorganischen Schädigungen sind spärlich.

\section{Schlussfolgerungen}

Aufgrund der großen Anzahl von Patienten mit Ganser-Syndrom bei denen eine hirnorganische Schädigung beschrieben wird und aufgrund der Betonung des Störungsmusters auf frontal-exekutiven Funktionen könnte man die Frage aufwerfen, ob alle Ganser-Patienten eine Schädigung des Frontalhirns aufweisen. Dem widerspricht die Tatsache, dass das Ganser-Syndrom als klinisches Bild sehr selten ist. Da bei unserer Patientin eindeutige Hinweise auf eine sehr untypische Repräsentation kognitiver Funktionen vorliegen, könnte man weiter fragen, ob Patienten mit Ganser-Syndrom eher eine untypische Repräsentation kognitiver Funktionen aufweisen, deren Schädigung dann das vielfältige psychopathologische Bild entstehen lassen. Diese These wird durch unseren Fall gestützt, müsste aber durch weitere differenzierte neuropsychologische Untersuchungen bei Patienten mit Ganser-Syndrom untermauert werden.

Die Literatur gibt darüber hinaus ein uneinheitliches Bild der neuropsychologischen und zerebralen Störungsmuster wieder, das nur vage schließen lässt, dass primär frontotemporale Funktionen und Bereiche bei Patienten mit Ganser-Syndrom geschädigt sind. Zieht man Modelle über dissoziative Störungen [19, 32] zur Erklärung heran, lässt sich eine ge- wisse Integration der Befunde erreichen, da die beschriebenen Störungsmuster wie auch die Psychopathologie des GanserSyndroms durch ein thalamokortikolimbisches Störungsmodell erklärt werden könnten.

Um diese Hypothese zu untermauern sollten Patienten mit Verdacht auf oder mit einem klar diagnostizierten GanserSyndrom unbedingt standardisiert neuropsychologisch und bildgebend untersucht werden und insbesondere sollte geprüft werden, inwiefern eine untypische Repräsentation kognitiver Funktionen vorliegt.

\section{Korrespondenzadresse \\ PD Dr. K. Gutbrod}

Abteilung für neuropsychologische

Rehabilitation, Neurologische Klinik der

Universität, Inselspital Bern

3010 Bern, Schweiz

klemens.gutrod@insel.ch

Interessenkonflikt. Der korrespondierende Autor gibt an, dass kein Interessenkonflikt besteht.

\section{Literatur}

1. Adler R, Touyz $S$ (1989) Ganser syndrome in a 10 year old boy-an 8 year follow up. Aust N Z J Psychiatry 23: 124-126

2. Alexander GE, DeLong MR, Strick PL (1986) Parallel organization of functionally segregated circuits linking basal ganglia and cortex. Annu Rev Neurosci 9: 357-381

3. Alexander MP (1997) Aphasia: clinical and anatomic aspects. In: Feinberg TE, Farah TE (eds) Behavioral neurology and neuropsychology. Mcgraw-Hill, New York, pp 133-149

4. Alexander MP, Annett M (1996) Crossed aphasia and related anomalies of cerebral organization: case reports and a genetic hypothesis. Brain Lang 55: 213-239

5. Andersen HS, Sestoft D, Lillebaek T (2001) Ganser syndrome after solitary confinement in prison: a short review and a case report. Nord J Psychiatry 55: 199-201

6. Apter A, Ratzoni G, lancu l et al. (1993) The Ganser syndrome in two adolescent brothers. J Am Acad Child Adolesc Psychiatry 32: 582-584

7. Assion H-J (2001) 100 Jahre Ganser-Syndrom ein Rückblick und Ausblick. In: Nissen G, Badura F (Hrsg) Schriftenreihe der Deutschen Gesellschaft für Geschichte der Nervenheilkunde. Bd. 7. Verlag Königshausen und Neumann, Würzburg

8. Assion H-J, Schmidt K (2004) Psychische Störungen der Schlitzohrigkeit? Zur Klassifikation des Ganser-Syndroms. In: Vollmoeller B (Hrsg) Grenzwertige psychische Störungen. Diagnostik und Therapie in Schwellenbereichen. Thieme, Stuttgart

9. Dabholkar PD (1987) Ganser syndrome. A case report and discussion. Br J Psychiatry 151: 256-258

10. Dalfen AK, Anthony F (2000) Head injury, dissociation and the Ganser syndrome. Brain Inj 14: 11011105 
Hier steht eine Anzeige.

算 Springer 
11. Damasio H (1981) Cerebral localization of aphasias. In: Sarno MT (ed) Acquired aphasia. Academic Press, New York

12. Dammann G (2004) Das Ganser Syndrom. In: Eckhardt-Henn A, Hoffmann SO (Hrsg) Dissoziative Bewusstseinsstörungen - Theorie, Symptomatik, Therapie. Schattauer, Stuttgart, S 188-194

13. Deibler MW, Hacker C, Rough J et al. (2003) Ganser's syndrome in a man with AIDS. Psychosomatics 44: 342-345

14. Dilling H, Mombour W, Schmidt MH (1999) Internationale Klassifikation psychischer Störungen ICD-10 Kapitel V (F). Hans Huber, Bern

15. Drechsler R (1997) Sprachstörungen nach Schädelhirntrauma. Gustav Narr, Tübingen

16. Ferstl EC, Cramon DY von (2005) Sprachverstehen im Kontext: Bildgebende Studien zu Kohärenz und Pragmatik. Sprache-Stimme-Gehör 29: 130-138

17. Fischer RS, Alexander MP, Gabriel C et al. (1991) Reversed lateralization of cognitive functions in right handers. Exceptions to classical aphasiology. Brain 114: 245-261

18. Freedman M, Alexander MP, Naeser MA (1984) Anatomic basis of transcortical motor aphasia. Neurology 34: 409-417

19. Frewen PA, Lanius RA (2006) Neurobiology of dissociation: unity and disunity in mind-body-brain. Psychiatr Clin North Am 29: 113-128

20. Fukatsu R, Fujii T, Yamadori A et al. (1997) Persisting childish behavior after bilateral thalamic infarcts. Eur Neurol 37: 230-235

21. Ganser S (1898) Ueber einen eigenartigen hysterischen Dämmerzustand. Arch Psychiat Nervenkr 30: 633-640

22. Glindemann R, Cramon DY von (1995) Kommunikationsstörungen bei Patienten mit Frontalhirnläsionen. Sprache-Stimme-Gehör 19: 1-17

23. Hampel H, Berger C, Muller N (1996) A case of Ganser's state presenting as a dementia syndrome. Psychopathology 29: 236-241

24. Härting C, Markowitsch HJ, Neufeld H et al. (2000) WMS-R. Wechsler Gedächtnistest - Revidierte Fassung. Manual. Hans Huber, Bern

25. Heron EA, Kritchevsky M, Delis DC (1991) Neuropsychological presentation of Ganser symptoms. J Clin Exp Neuropsychol 13: 652-666

26. Heubrock D, Petermann F (2000) Testbatterie zur Forensischen Neuropsychologie (TBFN): Neuropsychologische Diagnostik bei Simulationsverdacht. Testmanual. Swets \& Zeitlinger, Frankfurt

27. Huber W, Poeck K, Weniger D (2002) Aphasie. In: Hartje W, Poeck K (Hrsg) Klinische Neuropsychologie. Thieme, Stuttgart, S 80-144

28. Hugdahl K, Carlsson G, Uvebrant P, Lundervold AJ (1997) Dichotic-listening performance and intracarotid injections of amobarbital in children and adolescents. Preoperative and postoperative comparisons. Arch Neurol 54: 1494-1500

29. Hund-Georgiadis M, Zysset S, Weih K et al. (2001) Crossed nonaphasia in a dextral with left hemispheric lesions: a functional magnetic resonance imaging study of mirrored brain organization. Stroke 32: 2703-2707

30. Ladowsky-Brooks RL, Fischer CE (2003) Ganser symptoms in a case of frontal-temporal lobe dementia: is there a common neural substrate? J Clin Exp Neuropsychol 25: 761-768

31. Lang C, Dehm A, Dehm B, Leuschner T (1999) Kurze Aphasieprüfung. Handanweisung. Swets \& Zeitlinger, Frankfurt

32. Lanius RA, Bluhm R, Lanius U, Pain C (2006) A review of neuroimaging studies in PTSD: heterogeneity of response to symptom provocation. J Psychiatr Res 40: 709-729
33. Latcham R, White A, Sims A (1978) Ganser syndrome: the aetiological argument. J Neurol Neurosurg Psychiatry 41: 851-854

34. Lee HB, Koenig T (2001) A case of Ganser syndrome: organic or hysterical? Gen Hosp Psychiatry 23: $230-231$

35. Miller P, Bramble D, Buxton N (1997) Case study: Ganser syndrome in children and adolescents. J Am Acad Child Adolesc Psychiatry 36: 112-115

36. Mohr JP, Pessin MS, Finkelstein S et al. (1978) Broca aphasia: pathologic and clinical. Neurology 28: 311-324

37. Morris JC, Heyman A, Mohs RC et al. (1989) The Consortium to Establish a Registry for Alzheimer's Disease (CERAD). Part I. Clinical and neuropsychological assessment of Alzheimer's disease. Neurology 39: 1159-1165

38. Neau J-P, Bogousslavsky J (2001) Superficial middle cerebral artery syndromes. In: Bogousslavsky J, Caplan L (eds) Stroke syndromes. University Press, Cambridge, pp 405-427

39. Olbrich HM, Fritze J, Lanczik MH, Vauth R (1999) Schizophrenien und andere psychotische Störungen. In: Berger M (Hrsg) Psychiatrie und Psychotherapie. Urban und Fischer, München, S $405-$ 481

40. Prigatano GP, Roueche JT, Fordyce DJ (1986) Nonaphasic language disturbances after brain injury. In: Prigatano GP (ed) Neuropsychological rehabilitation after brain injury. John Hopkins University Press, Baltimore, MD, pp 18-28
41. Prutting CA, Kirchner D (1983) Applied pragmatics. In: Gallagher TM, Prutting CA (eds) Pragmatic assessment and intervention issues in language. College-Hill Press, San Diego, CA, pp 29-68

42. Pullicino $P$ (2001) Lenticulostriate arteries. In: Bogousslavsky J, Caplan L (eds) Stroke syndromes. University Press, Cambridge, pp 428-438

43. Refaat R, Firth DA, Robertson MM (2002) Uncomplicated Gilles de la Tourette syndrome and probable Ganser syndrome. A case report and review of the literature. Eur Child Adolesc Psychiatry 11: 234-239

44. Sass H, Wittchen H-U, Zaudig M, Houben I (2003) Diagnostische Kriterien DSM-IV-TR. Hogrefe, Göttingen

45. Schneider R, Klosinski G (1989) Travellers in five worlds: adolescents with Ganser's syndrome. Acta Paedopsychiatr 52: 150-155

46. Schnider A, Daniken C von, Gutbrod K (1996) The mechanisms of spontaneous and provoked confabulations. Brain 119: 1365-1375

47. Sigal M, Altmark D, Alfici S, Gelkopf M (1992) Ganser syndrome: a review of 15 cases. Compr Psychiatry $33: 134-138$

48. Spreen O, Strauss E (1998) A compendium of neuropsychological tests. Oxford Univeristy Press, New York

49. Tatu L, Moulin T, Bogousslavsky J, Duvernoy H (2001) Arterial territories of the human brain. In: Bogousslavsky J, Caplan L (eds) Stroke syndromes. University Press, Cambridge, pp 375-404

50. Tsoi WF (1973) The Ganser syndrome in Singapore: a report on ten cases. Br J Psychiatry 123: 567-572

51. Wittchen H-U, Zaudig M, Fydrich T (1997) SKID. Strukturiertes klinisches Interview für DSM-IV. Achse I und II. Hogrefe, Göttingen

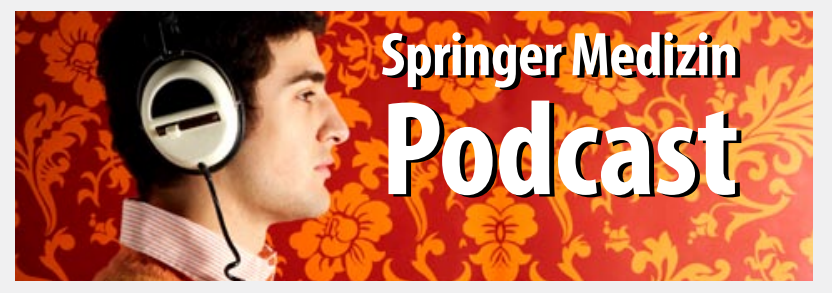

- Kongressnews

- Spannendes aus der Welt der Medizin

- Interviews Jeden Monat neu!

Jetzt kostenlos downloaden unter www.springer.de/podcast 


\section{Buchbesprechungen}

\section{H. Kornhuber, L. Deecke Wille und Gehirn}

Bielefeld, Locarno: Sirius 2007, 149 S., (ISBN 978-3-89528-628-5), 9.80 EUR

Die von Kornhuber ausformulierte Schrift ist in der von einer wegweisenden Entdeckung vorgegebenen Richtung aus der gemeinsam über Jahrzehnte fortgeführten Arbeit beider Autoren hervorgegangen. 1964/65 hatten Kornhuber als junger Dozent und Deecke als sein Doktorand auf der Suche nach neurophysiologischen Entsprechungen vor willentlichen Handlungen ein Bereitschaftspotential gefunden, das der frontomedial gelegenen motorischen Supplementärarea zugeordnet werden kann. Für die wissenschaftliche Psychologie spielte der Wille zu dieser Zeit, wie selbst noch in der 1980 erschienenen Monographie Heckhausens zur Motivation, keine Rolle. Erst in den 80er Jahren ist mit den aus der Neurophysiologie kommenden Anregungen, zu deren Beachtung international nicht zuletzt Popper und Eccles (1977) und die auf dem Bereitschaftspotential fußenden Experimente Libets (1985) beigetragen haben, die Willensthematik von Psychologen im Umkreis von Heckhausen und von Autoren wie Kuhl, Gollwitzer, Goschke, Walter und Goschke wieder aufgegriffen worden. Die Medien haben sie als Gegenstand eines Streites der Fachleute über die Willensfreiheit gerne zum Thema gemacht. Ein von $\mathrm{Ch}$. Geyer 2004 herausgegebener Sammelband „Hirnforschung und Willensfreiheit" lässt zusammen mit den Gegenstimmen den deterministischen Standpunkt zu Wort kommen, wie er sich pointiert in der Überschrift zu den Beiträgen der beiden ihn am entschiedensten vertretenen Neurobiologen äußert: „Wir sind determiniert. Die Hirnforschung befreit von Illusionen“ (G. Roth); , "Verschaltungen legen uns fest: Wir sollten aufhören, von Freiheit zu sprechen" (W. Singer). Die so schon in früheren Buchveröffentlichungen vertretene deterministische Position war eine Herausforderung für Kornhuber und Deecke. Zum gleichen Zeitpunkt wie ihr Buch und aus der psychiatrischen Erfahrung mit inm korrespondierend ist von $\mathrm{H}$.-L. Kröber in einem Handbuch der forensischen Psychiatrie der Artikel "Steuerungsfähigkeit und Willensfreiheit aus Psychiatrischer Sicht" erschienen. Er informiert über den aktuellen Stand der
Diskussion und greift bei der Beschäftigung mit der Willensthematik weit über das forensische Anliegen hinaus.

Die in diesem Kontext stehende Schrift hat den immer wieder anders und in anderen Grenzen verstandenen Willensbegriff sehr weit gefasst. Sie hat das dazu aus philosophischer, theologischer, psychologischer, neurowissenschaftlicher wie aus evolutionsbiologischer und menschenkundlicher Sicht Gesagte mit Ergebnissen eigener Forschung und den engagiert vorgetragenen eigenen Überzeugungen verknüpft, was bei der Fülle der Gesichtspunkte, für die schon die 16 Seiten Literatur bei rund einhundert Textseiten sprechen, auswählend und verdichtend geschehen musste. Ein in die historisch durchgehend zentrale Stellung des Gegenstandes einführender und von den Bemühungen großer Denker um den Willen vorgezeichneter Gang durch die Geistesgeschichte erreicht einen ersten Gipfel bereits in der griechischen Philosophie. Der von vielen Namen begleitete Weg führt über die Scholastik, die Renaissance, die Aufklärung, den deutschen Idealismus, Nietzsche, Jaspers, Max Scheeler, Nicolai Hartmann bis in die Gegenwart. Er wird bei den Philosophen, den Denkern des Ostens, der geisteswissenschaftlichen Psychologie, nicht aber für die von der spätjüdischen Tradition ausgehende eschatologische Richtung der Theologie, ganz überwiegend von Willensfreiheit bestimmt. Der experimentellen Psychologie ist der Wille nach Narziss Ach, Lindworski, Kurt Lewin unter dem Einfluss des Behaviorismus und des „Freudismus" verloren gegangen. Der Satz: „Nach dem Verschwinden des Willens aus der Psychologie ging der Neuanfang der Willensforschung von der Neurologie aus" leitet einen durch Abbildungen und ihre Legenden erläuterten Exkurs über die Experimente ein, die das Bereitschaftspotential mit seinen nicht nur für die Geschichte der Neurowissenschaften bedeutsamen Konsequenzen begründeten. Damit wird der eine Grundaspekt: Wille als eine „komplexe, umfassende Hirnfunktion" erreicht. Der Verfasser, der sich auch hier auf Arbeiten beider Autoren stützen kann, erläutert als Fachmann, und im einzelnen für den Fachmann, wie man sich die neurobiologische Fundierung dieser Komplexität vorzustellen hat. Der "Willenscortex“ muss, was im Grundsätzlichen schon Karl Kleist bei seinen Untersuchungen an Hirnverletzten des ersten Weltkrieges (1934) gesehen hatte, im Stirnhirn gesucht werden: die von geistigem Antrieb, Planung, Selbstführung, kreativem Denken bestimmte Komponente im dorsolateralen präfrontalen Cortex und im Frontalpol; Emotionskontrolle, Gesinnungen, gesínnungsgeleitetes ausdauerndes Handeln im orbitalen Cortex. Unter Berücksichtigung der Funktionen anderer Hirnzentren und der Verbindungen zu solchen Zentren kommt es entscheidend an auf die auf Rückbezogenheit beruhende Führungsrolle des frontalen Cortex. Der so fundierte Wille ist nicht der absolute freie Wille. Man könnte bei der von Kornhuber und Deecke verteidigten Willensfreiheit mit einer von Bieri in der philosophischen Argumentation gebrauchten Formulierung von bedingter Freiheit sprechen. Dem zweiten Grundaspekt: Wille als "Zentrum der Freiheit" könnte nur eine ausführliche Darstellung entsprechen, für die die ersten Sätze der Zusammenfassung eintreten sollen:„,Wille ist vernünftige Selbstführung des Menschen, ist Denken und Tun aus der Persönlichkeit und ihrem Kern, dem Selbst. Wille braucht Strategie, Ziele, Gründe, Methoden. Der Wille des Menschen ist kreativ und stützt sich auf Kultur und Zusammenarbeit. Er ist keine Automatik der Hirnphysiologie, sondern entsteht kulturell geprägt durch Lernen von Vorbildern, durch eigene Initiative, und durch Besinnung auf sich selbst ..." Wenn man einem Hinweis des Verfassers auf russische Kriegsgefangenschaft folgt und den Lebensdaten entnimmt, dass er als 17Jähriger in Gefangenschaft geraten sein muss und nicht vor 1949 freigekommen ist, wird man angeregt, nach dem 1961 erschienenen Handbuchbeitrag Kornhubers zu greifen, der in umfassender Darstellung „Psychologie und Psychopathologie der Kriegsgefangenschaft" behandelt. Man ahnt, dass es als prägende Erfahrung die Kräfte des Willens waren, die inn haben überleben lassen. So ist diese Schrift neben der Wissenschaft (mit mahnend eingestreuten ärztlich-therapeutischen Hinweisen) auch ein Bekenntnis.

Professor W. Janzarik (Heidelberg). 